TH2C-5

\title{
MEASURING COMPLEX PERMITTIVITY AND PERMEABILITY USING TIME DOMAIN NETWORK ANALYSIS
}

\author{
Jeffrey A. Jargon and Michael D. Janezic \\ National Institute of Standards and Technology \\ 325 Broadway, Mail Code 813.06, Boulder, CO 80303 USA \\ jargon@boulder.nist.gov, janezic@boulder.nist.gov
}

\begin{abstract}
We use a time domain network analyzer along with a recent improvement to the transmission/reflection method to determine the complex permittivity and permeability of a sample in a coaxial line. Our data show that accurate measurements can be made without a conventional frequency domain network analyzer.
\end{abstract}

\section{INTRODUCTION}

First proposed over twenty-five years ago [1], time domain network analysis (TDNA) is finally emerging as a practical, cost-effective alternative to traditional frequency domain network analysis (FDNA). Recent advances in instrumentation [2], calibration techniques [3], and optimization [4] have made TDNA a practical technique for a variety of implementations, including onwafer measurements [5].

In this paper, we apply TDNA to the determination of complex permittivity and permeability using an improved transmission/reflection (TR) method [6]. The TR technique applies a robust algorithm and avoids the illconditioning at frequencies corresponding to integral multiples of one-half wavelength in the sample. This method is best suited for high bandwidth measurements of materials with low to medium values of permittivity. Although time domain systems have been introduced in the past to characterize materials $[7,8]$, an affordable system that compares favorably to accurate FDNA has never been realized.

Our method applies to complex, frequency-dependent dielectric and magnetic materials. In contrast, simple time domain reflection (TDR) measurements provide at best only a frequency-independent estimate of the real part of the permittivity, and cannot handle materials in which both the permittivity and permeability are unknown.

To carry out the measurements, an unknown sample,

This publication is U.S. Government work and not protected by U.S, copyright. whose complex permittivity and permeability we wish to determine, is placed in a section of coaxial line and measured with a time domain network analyzer. Coaxial calibration standards are measured at the same test ports. Off-line, the waveform records of the samples and the calibration artifacts are converted to uncorrected frequency-dependent scattering parameters using a fast Fourier transform (FFT), and a calibration is performed. The calibrated scattering parameters of the unknown sample are then used to determine the complex permittivity and permeability.

We apply the multiline through-reflect-line (TRL) calibration [9] to TDNA measurements since this method permits error correction over a wide frequency band and fully characterizes the calibration lines.

\section{Calibration and Measurement}

We used a time domain network analyzer to collect data from the unknown samples and calibration artifacts. The system consists of a digital sampling oscilloscope (DSO) with time-domain reflection/transmission sample and source heads, and a personal computer for automated data acquisition, calibration, and calculation. Three software programs developed at the National Institute of Standards and Technology were used: one controls the DSO, acquires the time domain waveforms, and performs an FFT of the data; MultiCal implements multiline TRL and corrects for nonideal characteristic impedance [10]; and EPSMU determines the complex permittivity and permeability using the calibrated scattering parameters of the unknown sample.

For illustration, we chose a ferrite-loaded polymer (FLP) and a nickel-zinc ferrite (NZF). The FLP was $12.648 \mathrm{~mm}$ in length and the NZF was $5.626 \mathrm{~mm}$. Both materials were machined into $7 \mathrm{~mm}$ coaxial samples. Air gap corrections were not taken into account for this experiment, since we were concerned primarily about the relative comparisons between TDNA and FDNA. Each sample was placed at one end of a $10 \mathrm{~cm}$ long airline. 
For TRL standards, we used a set of commercially available APC-7 artifacts consisting of $2.25,10$, and 30 $\mathrm{cm}$ airlines, and a short circuit termination. We assumed that our sexless APC-7 connectors mated perfectly with our lines, allowing a direct connection between the two ports to serve as a thru line.

For each TDNA measurement, TDR and TDT records were collected at a sample density of $50 \mathrm{~ns}^{-1}$ using 1024 averages.

For the FDNA measurements, data were taken at frequencies ranging from $30 \mathrm{MHz}$ to $3 \mathrm{GHz}$ using 256 averages. Since our network analyzer contained only three samplers, we applied a two-tier calibration [11], the first tier being an open-short-load-through, and the second tier a multiline TRL calibration.

\section{RESULTS}

Figures 1-4 plot the real and imaginary parts of the permittivity and permeability of the FLP. The TDNA results compare favorably to the FDNA measurements, although there are some differences. The permittivity obtained from the TDNA measurements differs by up to $0.3+j 0.3$. The differences in permeability are limited to $0.1+j 0.1$ at most frequencies. Figures $5-8$ plot the permittivity and permeability of the NZF. The permittivity of this sample has a similar range of differences as the FLP, except at frequencies below $0.2 \mathrm{GHz}$ where differences approach $1.0+j 0.2$. The permeability differences are also relatively similar to those of the FLP but are hard to distinguish in the plots due to the wide range of permeability values.

\section{CONCLUSIONS}

In conjunction with the improved TR method and an accurate calibration scheme such as multiline TRL, TDNA is an accurate and cost-effective tool for determining complex permittivity and permeability. Our TDNA measurements of the real part of the permittivity and permeability showed agreement with FDNA on the order of $3 \%$ or better at most frequencies.

\section{ACKNOWLEDGMENTS}

The authors thank Roger Marks and James Baker-Jarvis for their guidance, Don DeGroot for his assistance with the TDNA measurements, and Sedki Riad for reviewing this manuscript.

\section{REFERENCES}

[1] A.M. Nicolson, "Broad-band microwave transmission characteristics from a single measurement of the transient response," IEEE Trans. Instrum. Meas., vol. 17, no. 4, pp. 395-402, December 1968.

[2] D.A. Smolyansky, S.K. Diamond, and V.K. Tripathi, "Accurate determination of the impedance profile using enhanced accuracy time domain reflection and transmission measurements," 44th ARFTG Conference, Boulder, CO, 1994.

[3] R.B. Marks, L.A. Hayden, J.A. Jargon, and F. Williams, "Time domain network analysis using the multiline TRL calibration," 44th ARFTG Conference, Boulder, CO 1994.

[4] D.C. DeGroot and R.B. Marks, "Optimizing timedomain network analysis," 46th ARFTG Conference, Scottsdale, AZ, 1995.

[5] R.B. Marks, D.C. DeGroot, and J.A. Jargon, "Highspeed interconnection using time domain network analysis," Advancing Microelectronics, vol. 22, November-December 1995.

[6] J. Baker-Jarvis, M.D. Janezic, J.H. Grosvenor, Jr., and R.G. Geyer, "Transmission/reflection and short-circuit line methods for measuring permittivity and permeability," Natl. Inst. Stand. Technol., Tech. Note 1355-R, 1993.

[7] A.M. Nicolson, "Measurement of the intrinsic properties of materials by time-domain techniques, "IEEE Trans. Instrum. Meas., vol. 19, no. 4, pp. 377-382, November 1970.

[8] T. Nguyen, G. Maze-Merceur, and G. Garat, "Microwave characterization of materials having high dielectric constant by use of time domain analysis," European Electromagnetics International Symposium, Bourdeaux, France, 1995.

[9] R.B. Marks, "A multiline method of network analyzer calibration," IEEE Trans. Microwave Theory Tech., vol. 39 , no. 7 , pp. 1205-1215, July 1991.

[10] R.B. Marks and D.F. Williams, "Characteristic impedance determination using propagation constant measurement," IEEE Microwave Guided Lett. 1, pp. 141143, June 1991.

[11] J.A. Jargon and R.B. Marks, "Two-tier multiline TRL for calibration of low-cost network analyzers," 46th ARFTG Conference, Scottsdale, AZ, 1995. 


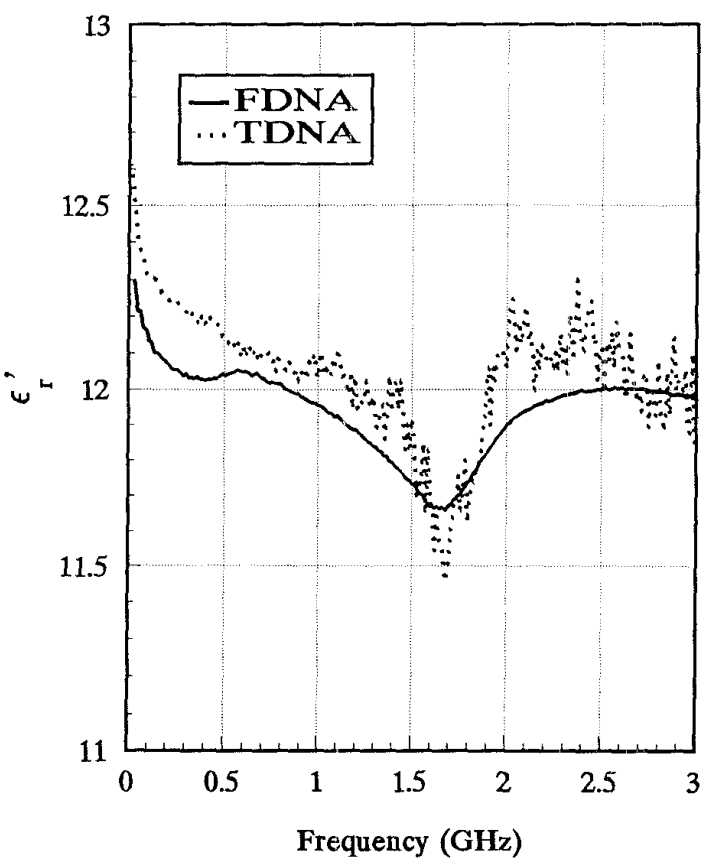

Fig. 1. Real part of permittivity of FLP.

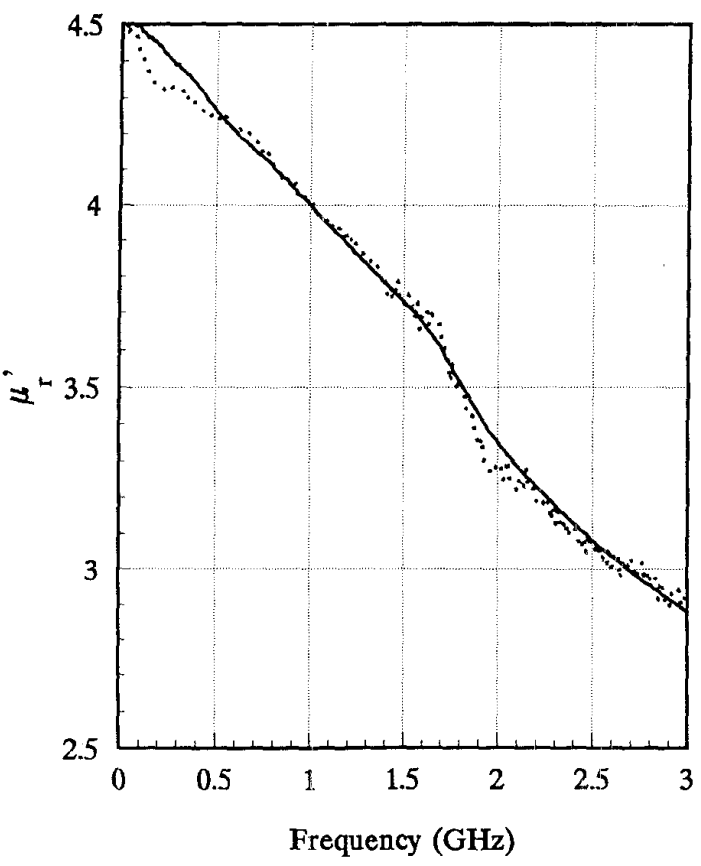

Fig. 3. Real part of permeability of FLP.

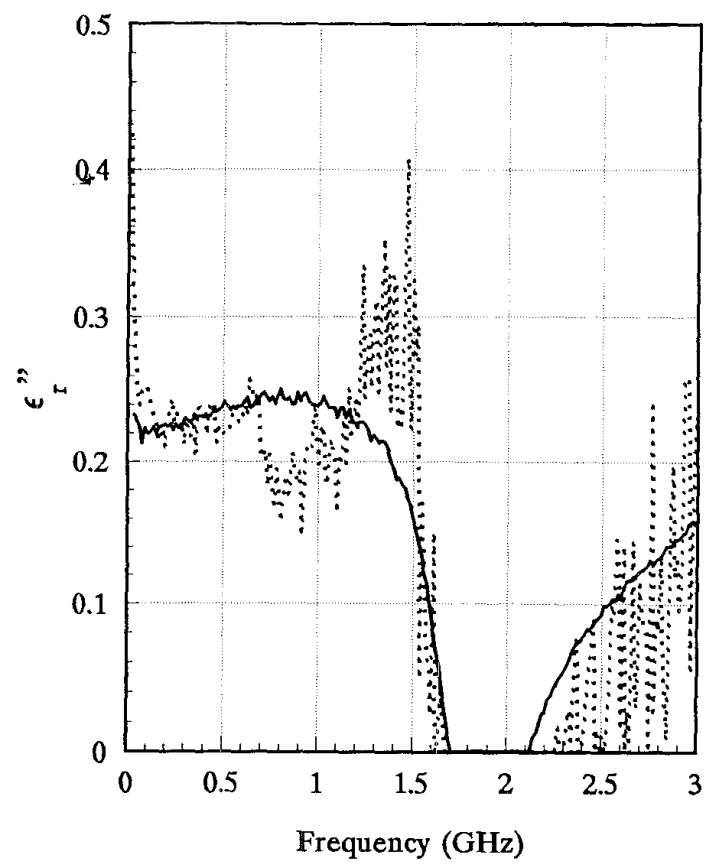

Fig. 2. Imaginary part of permittivity of FLP.

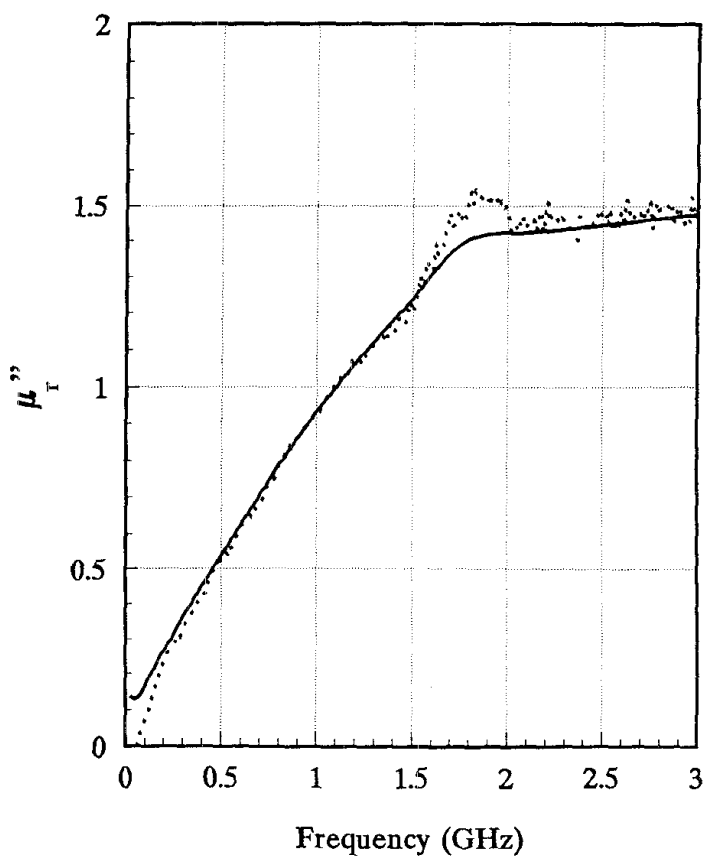

Fig. 4. Imaginary part of permeability of FLP. 


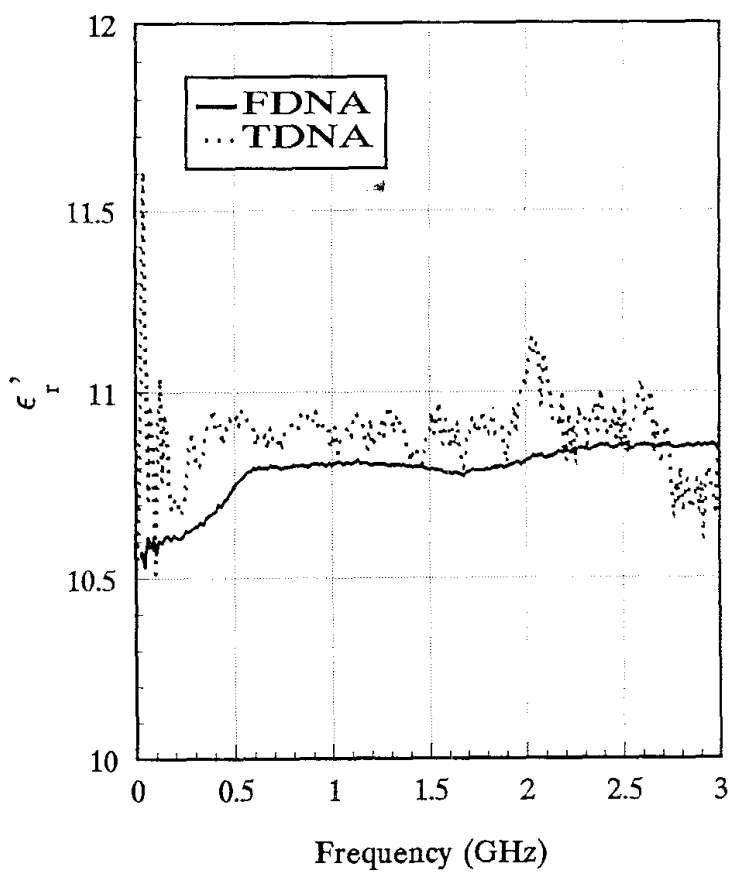

Fig. 5. Real part of permittivity of NZF.

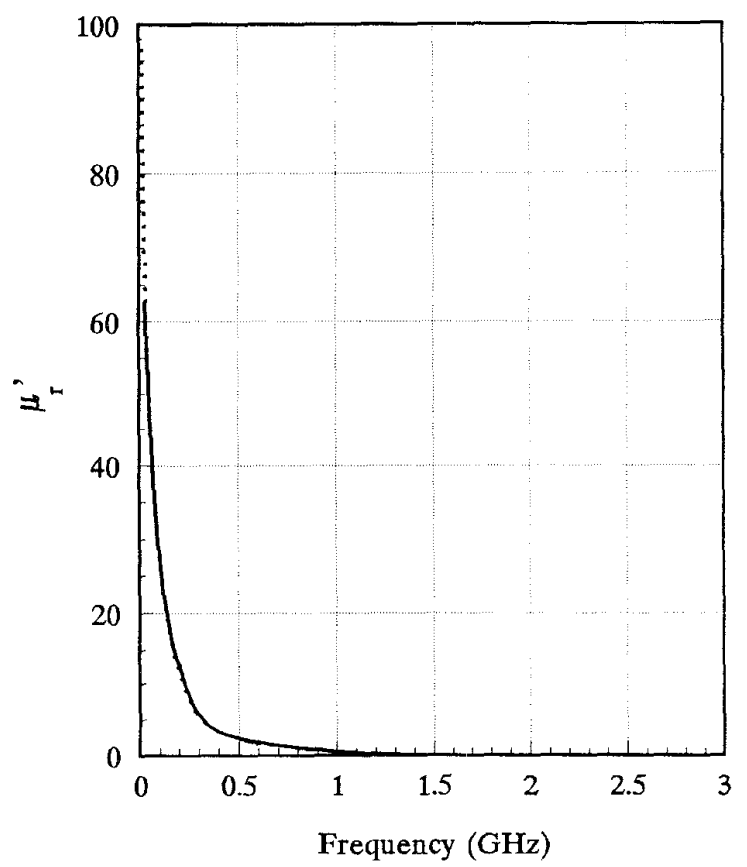

Fig. 7. Real part of permeability of NZF.

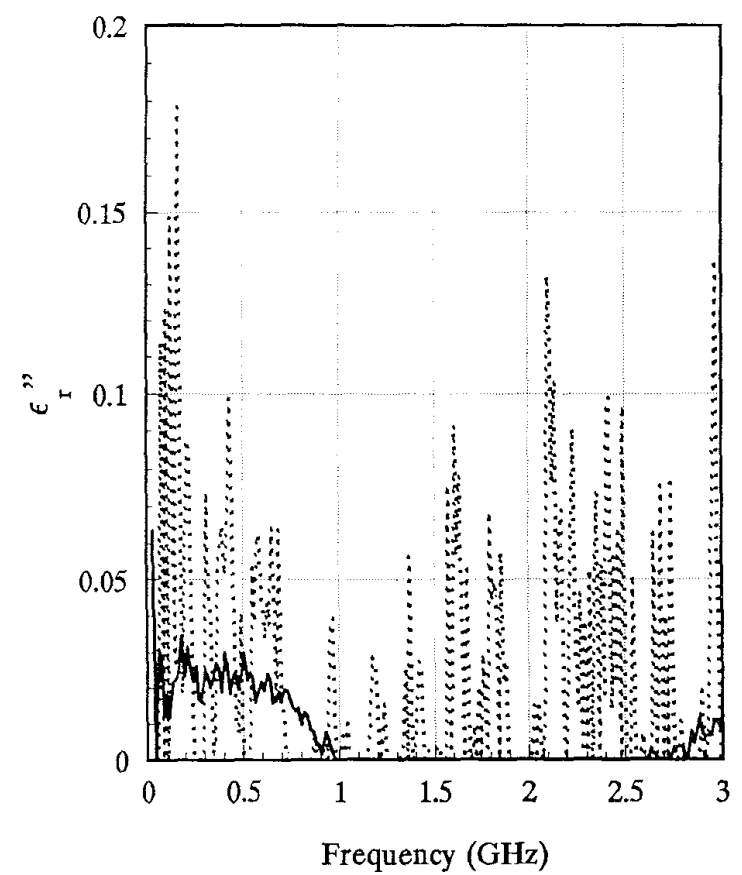

Fig. 6. Imaginary part of permittivity of NZF.

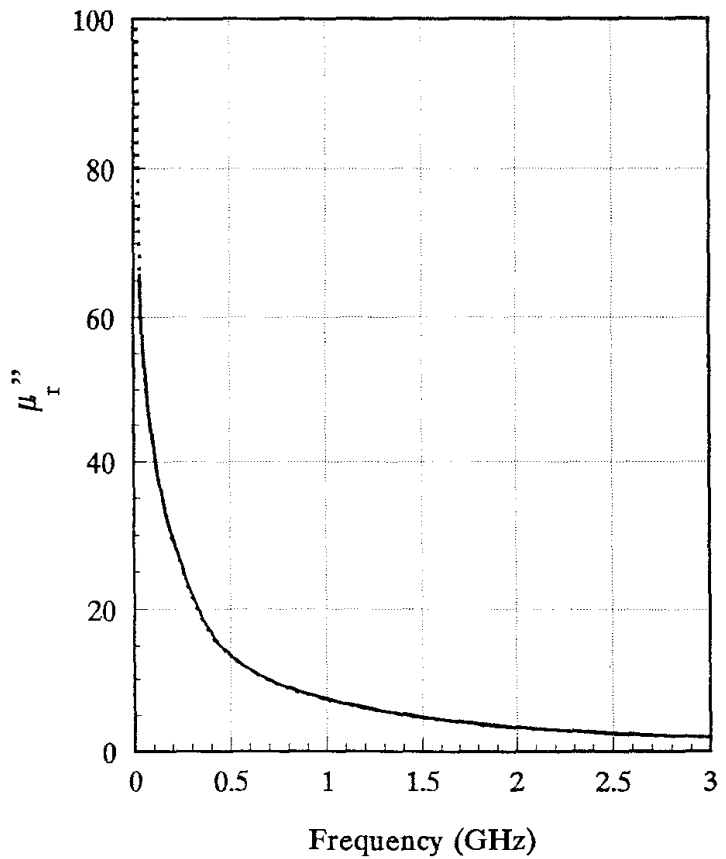

Fig. 8. Imaginary part of permeability of NZF. 